\title{
Fall Detection System based on BiLSTM Neural Network
}

\author{
${ }^{1}$ Biao Ye, ${ }^{2}$ Lasheng Yu \\ ${ }^{1,2}$ School of Computer science and Technology, Central South University, Changsha, Hunan, China; \\ 404871394@qq.com;yulasheng@csu.edu.cn
}

\begin{abstract}
The purpose of this article is to analyze the characteristics of human fall behavior to design a fall detection system. The existing fall detection algorithms have problems such as poor adaptability, single function and difficulty in processing large data and strong randomness. Therefore, a long-term and short-term memory recurrent neural network is used to improve the effect of falling behavior detection by exploring the internal correlation between sensor data. Firstly, the serialization representation method of sensor data, training data and detection input data is designed. The BiLSTM network has the characteristics of strong ability to sequence modeling and it is used to reduce the dimension of the data required by the fall detection model. then, the BiLSTM training algorithm for fall detection and the BiLSTM-based fall detection algorithm convert the fall detection into the classification problem of the input sequence; finally, the BiLSTM-based fall detection system was implemented on the Tensorflow platform. The detection and analysis of system were carried out using a bionic experiment data set which mimics a fall. The experimental results verify that the system can effectively improve the accuracy of fall detection to $90.47 \%$. At the same time, it can effectively detect the behavior of Near-falling, and help to take corresponding protective measures.
\end{abstract}

Keywords: BiLSTM neural network, fall detection; near-falling detection; sensor data

\section{Introduction}

With the advent of the ageing population, the number of old people who is living alone has also increased. As the age of the elderly increases, the muscle strength, balance, and responsiveness of the body gradually decline [1], which has increased the probability of falling behavior. Falling behavior is especially serious the lives and property of the elderly. It is very important to make timely warnings about possible fall behaviors, so as to reduce and avoid the harm caused. Current research at our country and other countries mainly includes: The old fall detection system based on ZigBee loT technology[2]. Visual-based fall detection system [3], and Wearable device-based fall detection system and other types.

Among them, the old man's fall detection which utilizing Zigbee technology in the Internet of Things is too single function, the use conditions are limited, and the visual fall detection involves the user's privacy and the camera can't be carried around, so the fall detection system based on the wearable device is easy to use and low in cost and become the current research hotspot.

The two most common sensors are the tilt angle sensors and the acceleration sensors. With the development of the gait recognition system, the behavior parameters of foot pressure are also applied to fall detection[4]. The foot pressure is measured by the force plate to measure the reaction force of the 
Biao Ye, Lasheng Yu; Fall Detection System based on BiLSTM Neural Network, Transactions on Machine Learning and Artificial I ntelligence, Volume 7 No 5 October, (2019); pp: 1-12

ground during walking. This paper synthesizes the changes of behavioral characteristics such as human body acceleration, tilt angle and foot pressure during daily activities and fall behaviors of human body. A fall detection algorithm combining multiple behavioral characteristics is proposed.

The threshold method is a commonly used fall detection algorithm. By setting one or more thresholds, the information such as acceleration and angular velocity acquired by the wearable device sensor is classified, and the main advantages are lower time and space complexityError! Reference source not found.. However, the threshold is mostly determined by experimental or empirical methods. The size of the threshold directly affects the recognition of the fall behavior, and there are problems such as poor applicability to different individuals.

As researchers deepen their focus on fall algorithms, they began to study hidden Markov chains[6], dynamic naive Bayesian networks[8], support vector machines [9], Multi-weight neural network [9], and $k$ nearest neighbors[11]. All of above based on the pattern recognition method applied to fall detection, these methods abstract the model data collected by the sensor for the classification of fall behavior, and have strong adaptability. However, only the sensor is used to obtain a single acceleration or some features for detection, lack of human behavior parameters, and there are problems such as insufficient precision, and the near-fall state with high use value is not distinguished. The neural network model has self-learning function, high-speed search for optimal solution, and association storage function compared with the traditional model. The neural network model can acquire a large amount of data through sensors to train, learn and mine the internal structural features of the data, and improve the adaptability and accuracy of the classification system. Relative to Convolution neural network spatially through the sliding window to store part of the historical input, Cyclic neural network can retain the previous input in time due to the inter-connected features of its hidden layers, by abstracting the association between the previous inputs, with a certain classification accuracy. However, since the previous input is continuously updated during the delivery process, the information retention rate is very low. LSTM joins the state in the hidden layer to support the defect of RNN, and solves the problem of gradient fading that is easy to appear in RNN.

At present, LSTM has been successfully used in language translation, image analysis, robot control, disease prediction and other fields.

This paper mainly introduces BiLSTM into the fall detection system. The main work arrangements are as follows:

(1) Firstly, the human behavior is classified, and analyzed the changes of the human body's gravity acceleration, angular acceleration and pressure division of the foot are analyzed.

(2) According to the characteristics of LSTM, the data acquired by the sensor is serialized and converted into an input sequence suitable for model training.

(3) With LSTM, historical information can be preserved, and the intrinsic relationship between location and pressure sensor data can be fully exploited by using feature discovery and abstract sequence intrinsic relationship. An iterative algorithm based on LSTM is designed to improve the effect of fall detection.

(4) On the TensorFlow platform, the LSTM-based iterative algorithm is used to implement the LSTMbased fall detection system. In addition to distinguishing between normal and fall behaviors, the existing fall detection system can accurately identify more dangerous. Close to the fall behavior.

(5) Obtain the sensor data on the experimenter's body through the bionic experiment, and use the data which has been pre-processed to train and test the fall detection model as the training and 
test data set. Compared with other algorithms on the same data set, the BiLSTM-based fall detection system can achieve higher accuracy and correctly detect the near-fall behavior, so as to better protect the fall behavior.

\section{Related Work}

\section{$2.1 \quad$ Fall Detection}

Vaidehi and his team, from Anna University in India, proposed a fall detection method based on static human image features. Through using the camera to collect pictures of the human body standing, falling, climbing and other actions, and extract the two aspects of the aspect ratio and the tilt angle of the human body from the picture, and perform the fall detection according to the threshold method [12]. Bogdan Kwolek and Michal Kepski use the Kinect sensor, which sets the threshold of the acceleration signal and filters out the number of non-falling events. Finally, by collecting the depth map and acceleration information as the data set, the k-nn algorithm is used to classified falls and non-fall events[13]. L.Chen,et al. used Bayesian network to realize human body attitude prediction. The attitude prediction combined with recognition algorithm was used to improve the prediction time. Then, the support vector machine method was used for fall recognition. The recall rate and accuracy rate reached $96.2 \%$ and $87.3 \%$ respectively. [14] D. Luo et al. proposed a fall detection algorithm based on random forest, which finally achieved $95.2 \%$ accuracy, $90.6 \%$ sensitivity and $93.5 \%$ specificity, and compared with SVM and back propagation (BP) neural networks. The conclusion of the detection algorithm. The pressure sensor-based fall detection system researched by Shi Xin et al. of Chongqing University analyzes the difference between the sole pressure in different human states through the system Error! Reference source not found..

\section{3 model based on LSTM neural network}

\subsection{LSTM neural network principle}

The LSTM neural network was first proposed by Hochreiter et al. [15], and then improved by Graves [17] is a kind of perfection based on RNN, which solves the problem of gradient extinction which is easy to appear in RNN. A single RNN hidden layer unit is shown in Figure 1.

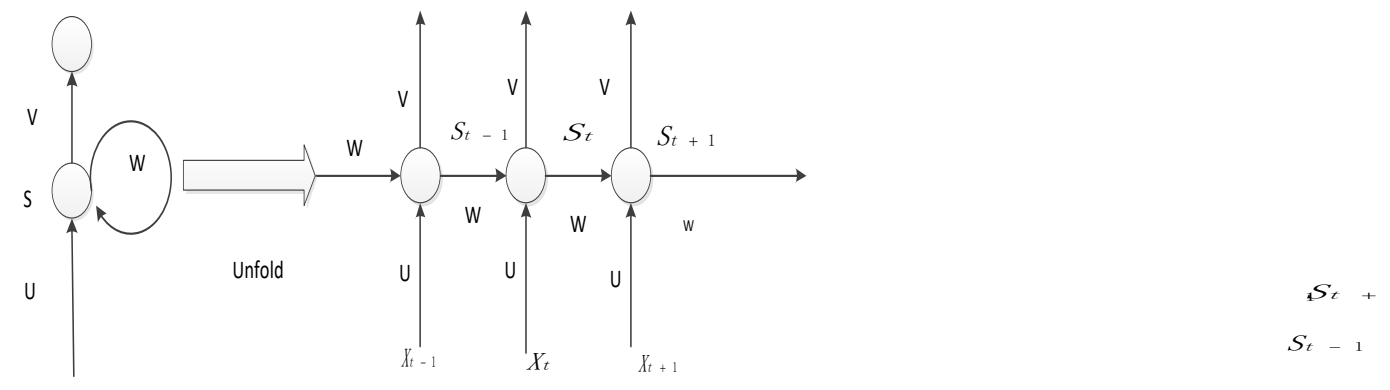

Figure 1: Single RNN neuron expansion diagram

As shown, $U, V$, and $W$ are the input to the hidden layer, the hidden layer to the hidden layer, the hidden layer to the output weight, $x$ is the input of the neural network, $O$ is the output of the neural network, and $S$ is the current state of the moment in the hidden layer. In traditional neural networks, the parameters of each network layer are unshared. In RNN, each step shares each parameter $U, V, W$. This reflects that every step in the RNN is doing the same thing, but the input is different. This training method 
greatly reduces the parameters that need to be learned in the network, and greatly shortens the training time under the premise of ensuring accuracy.

However, for the standard RNN architecture, the "context" that can be connected in practice is very limited, and the influence of "memory" at the far end on the output is either small or exponential. LSTM is an improved RNN for solving the phenomenon of gradient demise. LSTM unit is shown in Figure 2.

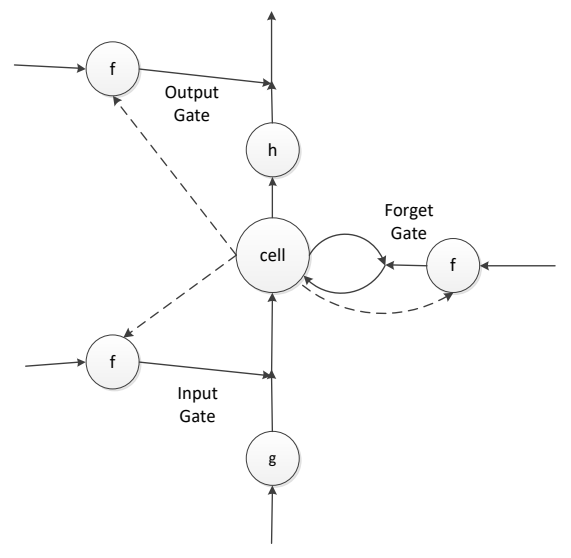

Figure 2: Single LSTM neuron

Algorithms implemented in LSTM neurons include:

$$
\begin{array}{cc}
i_{t}=\sigma\left(W_{t} x_{t}+U_{i} h_{t-1}+b_{i}\right) & f_{t}=\sigma\left(W_{f} x_{t}+U_{f} h_{t-1}+b_{f}\right) \\
\widetilde{c_{t}}=\tan h\left(W_{c} x_{t}+U_{c} h_{t-1}+b_{c}\right) & c_{t}=f_{t} \odot c_{t-1}+i_{t} \odot \widetilde{c_{t}} \\
o_{t}=\sigma\left(W_{o} x_{t}+U_{o} h_{t-1}+b_{o}\right) & h_{t}=o_{t} \odot \tan h\left(c_{t}\right)
\end{array}
$$

Among them, $x_{t}$ represents the input vector of time $t, b$ represents the offset value, $i_{t}$ represents the input gate which determines how much of the network input is retained to the cell state at the current time, $\mathrm{f}_{\mathrm{t}}$ represents that the forgetting gate determines how much the state of the neural unit remains to the current state of the neural unit at the last moment, $\mathrm{O}_{\mathrm{t}}$ represents the output gate, which controls how much the unit state has output to $\mathrm{h}_{\mathrm{t}}, \widetilde{\mathrm{c}_{\mathrm{t}}}$ is used to describe the current input neural unit status, $\mathrm{C}_{\mathrm{t}}$ represent the state of the nerve unit at the current time, $U$ and $\mathrm{W}$ represent weight matrix, $\tan h$ represents a hyperbolic tangent activation function that processes the input data to map it to the range $[-1,1], \sigma$ represents that the sigmoid function used on the three middle gates maps the input to $[0,1]$, $h_{t}$ represents the output of the neural unit signal at time $t$.

In addition to the detection of the fall state and the non-fall state, the fall detection model in this paper adds a very research-worthy state close to the fall. The normal LSTM is a one-way neural network that can only be saved from the back and forth. The status information is transferred, and the relationship between the incoming state and the current state cannot be discovered. Therefore, this design uses BiLSTMs with opposite directions to fully exploit the relationship between two adjacent time state sequences. The structure is shown in Figure 3. 


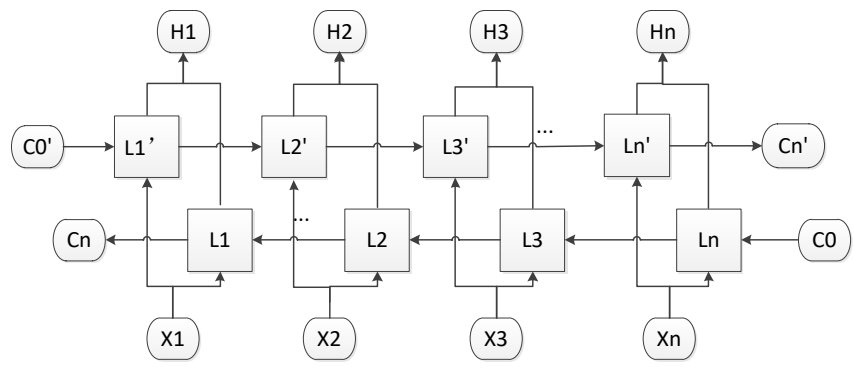

Figure 3: BiLSTM structure

As shown in the figure, in the forward transfer, the $\mathrm{Cn}$ of the hidden layer is related to $\mathrm{Cn}-1$; in the reverse calculation, $\mathrm{Cn}$ is related to $\mathrm{Cn}+1$ :

$$
\begin{aligned}
& C n=f\left(U_{1} x_{n}+W_{1^{c} n-1}\right) \quad C_{n}^{\prime}=f\left(U_{2} x_{t}+W_{2} C_{n+1}^{\prime}\right) \\
& O_{t}=g\left(V C_{n}+V^{\prime} C_{n}^{\prime}\right)
\end{aligned}
$$

\subsection{Sensor data processing}

\subsubsection{Acquisition of acceleration and angular velocity}

The module used for the combined acceleration and angular velocity acquisition required in this paper is the MPU6050, which has an integrated 6-axis motion processing component. The MPU6050 not only integrates a three-axis accelerometer and a three-axis gyroscope, In the MPU6050, the signals collected by accelerometers and gyroscopes are all implemented by three 16-bit analog-to-digital converters. The conversion outputs the result in digital form. To accurately track fast and slow motion, the sensor's measurement range is user-controllable. Among them, the range of the gyroscope can be divided into: $\pm 250^{\circ} / \mathrm{sec}, \pm 500^{\circ} / \mathrm{sec}, \pm 10007 \mathrm{sec}, \pm 2000^{\circ} / \mathrm{sec}$. Acceleration measurement range from small to large: $\pm 2 \mathrm{~g}, \pm 4 \mathrm{~g}, \pm 8 \mathrm{~g}, \pm 16 \mathrm{~g}$.

\subsubsection{Acquisition of plantar pressure}

When the movement of the human body changes, the pressure of the sole also sends a series of changes. The pressure insole used in this paper puts the pressure sensor in the insole and the shoe, and can measure the pressure of the sole in real time.
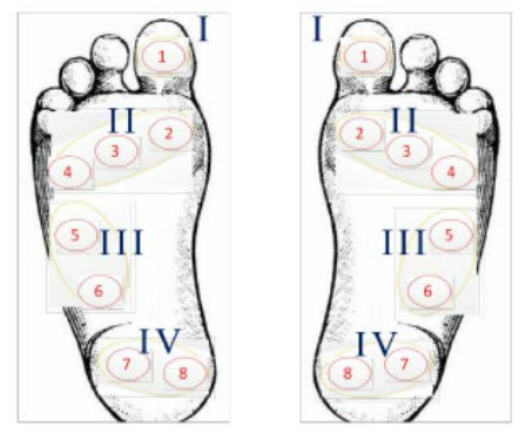

Figure 4: Two-leg pressure sensor distribution diagram

1 to 8 in Figure 4 are respectively equipped with a pressure sensor (Force Sensitive Resistor, FSR). The left and right feet ( $L$ for the left foot and $R$ for the right foot) are divided into the same way, forming a symmetrical area in the plane. Region I have one FSR No. 1 and is located in the thumb; Region II has FSRs 2, 3, and 4 located in the plantar tibia; Region III has FSRs 5 and 6 on the lateral side of the foot; Region IV 
has FSRs No. 7 and No. 8 Heel. According to the information of 150 sampling points collected by the plantar sensor, the voltage output value converted from the plantar pressure changes regularly during normal walking, and periodically changes from $0 \mathrm{~V}$ to $3.3 \mathrm{~V}$.

\subsubsection{Data serialization}

When we only use $\vec{a}=\left\{a_{x}(t), a_{y}(t), a_{z}(t)\right\}$ to represent three axial acceleration data, the acceleration data of one axis alone is not enough to accurately determine the posture of the human body. Therefore, SMV (Signal Magnitude Vector) is introduced as a characteristic parameter to describe the motion state of the human body. this parameter can reflect the severity of human exercise very well.

Some documents also refer to it as signal vector mode (SVM) and can be divided into $S M V_{A}$ and. $S M V_{W}$. Among them, $S M V_{A}$ represents the space triaxial acceleration vector sum, and some documents are also called the combined acceleration, and the calculation formula of the acceleration at a certain time $t$ :

The formula for calculating the angular velocity at a certain time:

$$
S M V_{w}=\sqrt{\omega_{x}^{2}(t)+\omega_{y}^{2}(t)+\omega_{z}^{2}(t)}
$$

The rate of change of acceleration SMA indicates the speed of acceleration change, and also reflects the degree of action change. The calculation formula:

$$
\mathrm{SMA}=\frac{1}{t}\left(\int_{0}^{t}\left|a_{x}(\mathrm{t})\right| \mathrm{d} t+\int_{0}^{t}\left|a_{y}(\mathrm{t})\right| \mathrm{d} t+\int_{0}^{t}\left|a_{z}(\mathrm{t})\right| \mathrm{d} t\right)
$$

Combined acceleration rate MADS (mean absolute value of different $S M V_{A}$ )

It is an important parameter that distinguishes the state of motion of the human body. The calculation formula is:

$$
M A D S=\frac{1}{T} \int_{0}^{T}\left|S M V_{A}^{\prime}\right| \mathrm{d} t
$$

The human body tilt angle is defined as the human torso (Z-axis positive) and vertical upward

The angle between the calculation formula:

$$
B T A=\arccos \left(\frac{a_{2}(t)}{g}\right) \times\left(\frac{180}{\pi}\right)
$$

The position sensor data sequence SS is defined, which includes three values of the $S M A, S M V_{A}$ and $B T A$ obtained by the gravity acceleration and angular acceleration, which converts the spatial position information collected by the position sensor into a data sequence, Then define the pressure wear sensor data sequence, PS1 contains the pressure values L1 L8 obtained by the left foot pressure sensor, PS2 contains R1 R8, then merge them and define the behavior training array AS (SS, PS1, PS2, action), action The type of current behavior, 0 means non-falling, 1 means falling.

LSTM can discover and abstract the relationship between each unit in the input sequence, and obtain the abstraction of the relationship between multiple position sensors, which provides a basis for improving the accuracy and adaptability of fall detection. 
In AS, the 3 values in each SS included in the AS and the values of PS1, PS2, and action are used as independent input units, and then the independent input unit is used as the input of the input layer for the training of the LSTM for fall detection. The output layer calculates the current probability Pt of the behavior training sequence group and counts the total probability P of each AS as the basis for training and detection.

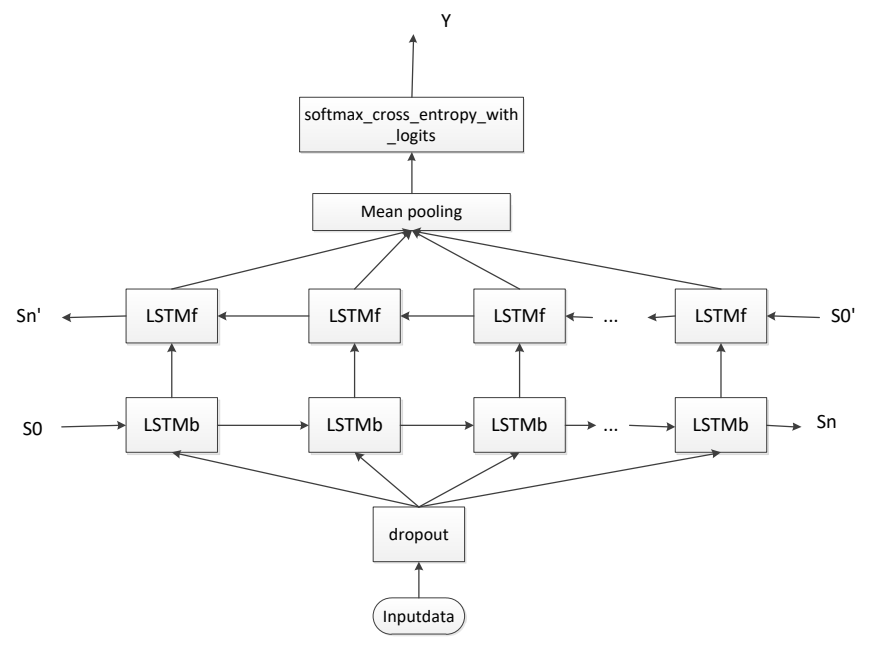

Figure5: Fall detection model

The Tensorflow flow chart of the research build is shown in Figure 5. in the model diagram, Input data represents an instance of a person's motion state at a certain moment, that is, a multivariate time series. The dropout operation randomly picks out the neural units that work together. The purpose is to prevent the model from being over-fitting, eliminating the weakening of the joint adaptability between the neuron nodes and enhancing the generalization ability. Next, the data is passed into two identically structured LSTMs, a forward input sequence, and an inverted input sequence, which combine the outputs of the two as the final result.

On the last layer of the BiLSTM neural network, the Mean Pooling mechanism can be used to fuse the result information of each node of the LSTM network. The weighted average of the output results is obtained by superimposing the output contents of the respective nodes of the BiLSTM and dividing by the number of input nodes. The weighted average of the output results is obtained by superimposing the output contents of the respective nodes of the BiLSTM and dividing by the number of input nodes. After the softmax activation function, the classification problem is transformed into a probability problem by mapping each output to a 0 to 1 interval.

The cross entropy loss function is used to calculate the error between the model output and the real category label.

The above is the BiLSTM-based fall detection model built using TensorFlow. The main training parameters of this model include the skew and weight between the input layer and the hidden layer, and the weights and offset values of the three gates in the LSTM neuron unit. After the loss function Loss is given, the training operation provided by TensorFlow can automatically find the differential derivative of Loss on each parameter and train the model by gradient descent method. 


\section{Experiment and Analysis}

this experiment collected 8 females and 12 males in the 18-40 age group. For the 20 samples, the mean and variance of their age, height and weight were $(26.2 \pm 6.7)$ years, $(165.2 \pm 5.8) \mathrm{cm}$ and $(70.8 \pm 15.1) \mathrm{kg}$, respectively.

The action of the specific experiment is shown in Table 5.1

Table 5.1 Action in the Simulation experiment

\begin{tabular}{|c|c|c|}
\hline Action & Category & \multirow{2}{*}{ Number of experiments } \\
\hline Walk & Non-falling behavior & \\
\hline Bend over & Non-falling behavior & \\
\hline Underarm & Non-falling behavior & \multirow{2}{*}{10 times / person } \\
\hline Stand up & Non-falling behavior & \\
\hline Lean forward & Near-fall behavior & \\
\hline Tilt back & Near-fall behavior & \\
\hline Dump forward & Fall behavior & \\
\hline Dump backwards & Fall behavior & \\
\hline Slip to the left & Fall behavior & Fall behavior \\
\hline Slip to the right & &
\end{tabular}

According to the information in the above table, the experimenter simulates the three periods of non-fall, near fall, and fall in the daily life of the elderly.

Perform 10 experiments in each state for a total of 200 experiments to meet the training and testing requirements of the BiLSTM model.

When constructing the model training set, this paper selects 4 males and 1 female as samples. The specific information is shown in Table 5.2.

Table 5.2 Training data set

\begin{tabular}{|c|c|l|c|c|}
\hline Sample & Gender & Age & Height & Weight \\
\hline Sample 1 & Male & 30 years old & $168 \mathrm{~cm}$ & $79.6 \mathrm{~kg}$ \\
\hline Sample 2 & Male & 22 years old & $176 \mathrm{~cm}$ & $75 \mathrm{~kg}$ \\
\hline Sample 3 & Male & 26 years old & $171 \mathrm{~cm}$ & $67 \mathrm{~kg}$ \\
\hline Sample 4 & Male & 28 years old & $162 \mathrm{~cm}$ & $70 \mathrm{~kg}$ \\
\hline Sample 5 & Female & 24 years old & $168 \mathrm{~cm}$ & $55 \mathrm{~kg}$ \\
\hline
\end{tabular}

Training Set of Fall Detection Model Each behavior training sequence group contains 8 pressure sensor data, 1 acceleration sensor data, 1 angular acceleration sensor data, and one status information. The remaining 15 samples were selected from 105 sensor state data to construct 5 test data sets for model testing, a total of 210 behavioral detection sequences.

The input sequence data includes 8 pressure sensor data and 1 acceleration sensor data and 1 angular acceleration sensor data, so the input node of the model can be set to 11 . The output layer nodes are set to three, corresponding to the categories of the three types of human fall states. Related parameters are in Table 5.3. 
Table 5.3 Related parameters

\begin{tabular}{|c|c|}
\hline Parameter name & Parameter value \\
\hline Number of input layer nodes & 3 \\
\hline Number of output layer nodes & 1 \\
\hline The number of layers in the hidden layer & 128 \\
\hline Number of hidden layer nodes per layer & $1 \mathrm{e}^{-4}$ \\
\hline Learning rate & 5 \\
\hline Number of instances per training & 1 \\
\hline Forgive_bias & .1 \\
\hline
\end{tabular}

Here we use the method called random_uniform_initialize provided by TensorFlow to initialize the training parameters of the logistic regression layer, We also used the method called orthogonal_initializer to initialize the parameters of the forgetting gate, input gate, and output gate in LSTMCell。Also, set the parameter Forget_bias to 1.0 when creating a new LSTMCell. When set the parameter Forget_bias to 1.0, the LSTMCell will not pass any state, The initial value of the learning rate is set to $1 \mathrm{e}^{-4}$, and the learning rate is adjusted by using the AdaDelta method in TensorFlow. The number of hidden layer nodes is set to 128.

The quality of a fall detection algorithm can usually be judged by three indicators: Accuracy, Precision and Recall.

TP: The number of times a fall behavior is predicted as a fall behavior

FP: The number of times a non-fall behavior is predicted as a fall behavior

FN: The number of times a fall behavior is predicted to be non-falling

TN: The number of times a non-fall is predicted to be non-falling

Precision: $\mathrm{TP} /(\mathrm{TP}+\mathrm{FP})$

Accuracy: $(T P+T N) /(T P+F N+F P+T N)$

Recall: $T P /(T P+F N)$

Table 5.4 Test results of BiLSTM detection of fall behavior

\begin{tabular}{|c|l|l|l|l|l|l|c|}
\hline Test set & TP & TN & FP & FN & Accuracy & Precision & Recall \\
\hline Test set 1 & 18 & 9 & 1 & 1 & $94.73 \%$ & $93.10 \%$ & $94.73 \%$ \\
\hline Test set 2 & 10 & 6 & 2 & 0 & $88.89 \%$ & $83.33 \%$ & $100 \%$ \\
\hline Test set 3 & 12 & 7 & 2 & 0 & $89.47 \%$ & $85.71 \%$ & $100 \%$ \\
\hline Test set 4 & 12 & 3 & 1 & 0 & $93.75 \%$ & $92.30 \%$ & $100 \%$ \\
\hline Test set 5 & 14 & 4 & 1 & 1 & $90.00 \%$ & $93.33 \%$ & $93.33 \%$ \\
\hline Total & 66 & 29 & 7 & 3 & $90.47 \%$ & $90.4 \%$ & $95.65 \%$ \\
\hline
\end{tabular}


Table 5.5 Test results of Near-fall detected by BiLSTM

\begin{tabular}{|c|c|c|c|c|}
\hline Test set & $\begin{array}{c}\text { Total behaviour } \\
\text { sequence }\end{array}$ & Near-fall sequence & Number of the correct Prediction & Accuracy \\
\hline Test set 1 & 58 & 6 & 5 & $83.33 \%$ \\
\hline Test set 2 & 36 & 4 & 4 & $100 \%$ \\
\hline Test set 3 & 42 & 4 & 3 & $100 \%$ \\
\hline Test set 4 & 32 & 3 & 4 & $100 \%$ \\
\hline Test set 5 & 40 & 5 & 19 & $80 \%$ \\
\hline Total & 132 & 22 & $86.36 \%$ \\
\hline
\end{tabular}

loss

tag: loss/loss

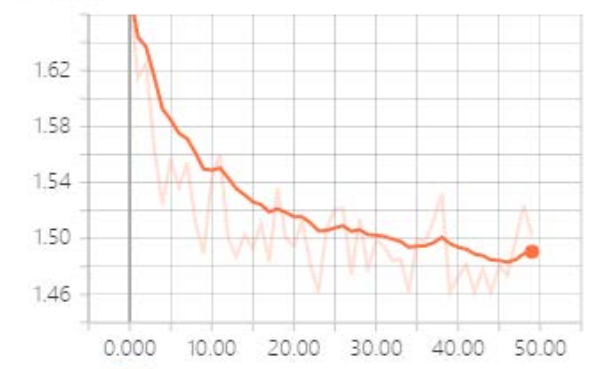

I]

Figure 7: the loss function value of the BiLSTM

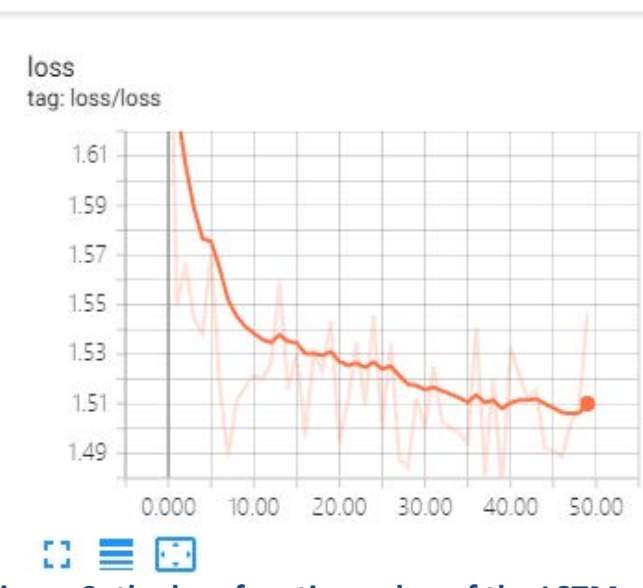

Figure 9: the loss function value of the LSTM

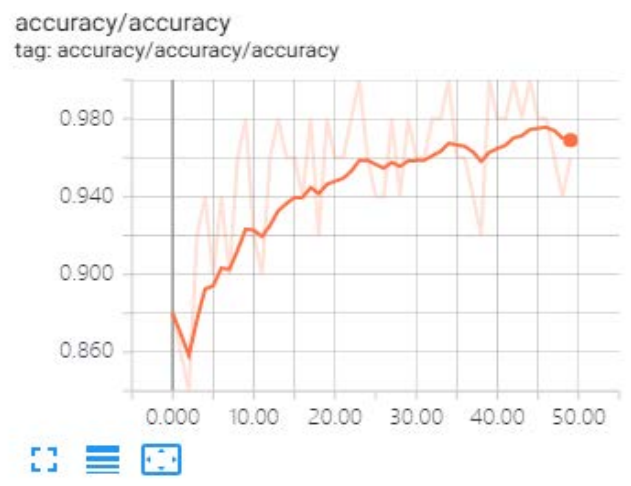

Figure8: Curve of BiLSTM classification accuracy rate accuracy/accuracy tag: accuracy/accuracy/accuracy

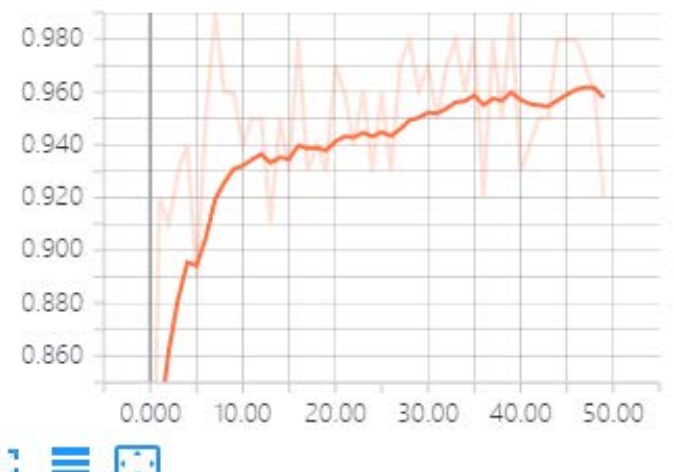

Figure 10: Curve of LSTM classification accuracy rate

Table 5.6 Compared with other fall detection algorithms

\begin{tabular}{|c|c|c|c|}
\hline Algorithms & Accuracy & Precision & Recall \\
\hline SVM & $87.45 \%$ & $86.9 \%$ & $89.71 \%$ \\
\hline LSTM & $88.1 \%$ & $88.92 \%$ & $90.23 \%$ \\
\hline BiLSTM & $90.47 \%$ & $90.4 \%$ & $95.65 \%$ \\
\hline
\end{tabular}




\section{Conclusion}

As we can be seen from the above tables and figures, the BiLSTM has significantly improved the Accuracy, Precision, and Recall rate compared with other algorithms, indicating that the BiLSTM can be better explored and abstracted the relationship between the sensor sequence data and the state can better judge the fall behavior and more valuable near-fall behavior. With the increase of training data and enrichment and further optimization of the algorithm, the detection model can be more perfect.

\section{REFERENCES}

[1] Mengyu Wu, Weihua Yu, Qian Ge, Cai Xuan, Jinping Qian, Ziwei Wang. Meta-analysis of the effects of exercise intervention on the fall, gait and balance of debilitated elderly [J]. Evidence-based nursing, 2018, 4 (11): 966-972 (in Chinese)

[2] Yu Zheng. Design and implementation of community elderly fall monitoring system based on ZigBee and Ethernet [D]. South China University of Technology, 2018. (in Chinese)

[3] Yuan Jie. Vision-based design and research of fall detection system for the elderly [D]. Jiangxi University of Technology, 2018. (in Chinese)

[4] Ding Yafei. Design of Fall Detection and Remote Monitoring System Based on Wearable Devices[J]. Information and Computer (Theoretical Edition), 2018(04) (in Chinese)

[5] Shi Xin. Research on motion human body behavior recognition based on pressure-sensing gait[D]. Chongqing University, 2010. (in Chinese)

[6] Niu Dezhen, Liu Yawen, Cai Tao, Peng Changsheng, Zhan Yongzhao, Liang Jun. A fall detection system based on recurrent neural network[J]. Journal of Intelligent Systems, 2018, 13(03): 380-387. (in Chinese)

[7] FANG-YIE LEU, CHIA-YIN KO, YI-CHEN LIN, et al.Smart Sensors Networks[M]. United Kingdom: Mara Conner, 2017: 205-237

[8] CHEN L, MA H T, LIU S, et al. Posture estimation by Bayesian Network with Belief Propagation[C]//TENCON 2013-2013 IEEE Region 10 Conference. Xi'an, China,2013: 1-4.

[9] Duan K B, Keerthi S S. Which is the best multiclass SVM method? An empirical study[M]// Multiple Classifier Systems. Springer Berlin Heidelberg, 2005:278-285.

[10] Zhai Yujian,Hua Liang,Chen Ling,Gu Juping,Shen Wei.Study on Intelligent Recognition of Falling Old Man Based on Multi-weight Neural Network[J].Science and Technology and Engineering,2015,15(04).(in Chinese)

[11] SZ Erdogan, TT Bilgin, J Cho. Fall-down detection by using K-nearest neighbor algorithm on WSN data[C]//GLOBECOM Workshops. Houston, USA, 2011:2054-2058.

[12] Vaidehi V, Ganapathy K, Mohan K, et al. Video based automatic fall detection in indoor environment [C].In: Proceeding of International Conference on Recent Trends in Information Technology, 2011:1016-1020. 
Biao Ye, Lasheng Yu; Fall Detection System based on BiLSTM Neural Network, Transactions on Machine Learning and Artificial Intelligence, Volume 7 No 5 October, (2019); pp: 1-12

[13] Kwolek B , Kepski M . Improving fall detection by the use of depth sensor and accelerometer[M]. Elsevier Science Publishers B. V. 2015.

[14] LUO D, LUO H, SCHOOL I. Fall-down detection algorithm based on random forest[J]. Journal of computer applications, 2015, 35(11): 3157-3160.

[15] Shi Xin. Research on motion human body behavior recognition based on pressure-sensing gait[D]. Chongqing University, 2010. (in Chinese)

[16] HOCHREITER S, SEHMIDHUBERJ. Long short-term memory[J]. Neural Computation, 1997, 9(8): 1735-1780.

[17] GRAVES A. Supervised sequence labelling with recurrent neural networks[D]. Ph. D Dissertation. Manno. Switzerland: Technical University of Munich, 2008. 